\title{
Surgical treatment of a giant unruptured left sinus of Valsava aneurysm requiring coronary artery bypass grafting
}

\author{
Panagiota Anna Chousou MD MRCP, Leisa Freeman MBChB FRCP FESC FHEA
}

\begin{abstract}
PA Chousou, L Freeman. Surgical treatment of a giant unruptured left sinus of Valsava aneurysm requiring coronary artery bypass grafting. Curr Res Cardiol 2015;2(1):45-46.
\end{abstract}

The present article describes a case involving a 74-year-old woman who presented with atypical chest pain and was found to have a giant aneurysm of the left sinus of Valsalva. Computed tomography coronary angiography performed before surgery showed a left main stem arising from the superior aspect of the left coronary sinus (stretched superiorly over, but not within, the aneurysm sac). The patient underwent surgery and an aortic valve replacement and root graft were performed. Intraoperatively, there was a

\section{CASE PRESENTATION}

A 74-year-old woman presented to the Rapid Access Chest Pain Clinic at Norfolk and Norwich University Hospital (Norwich, United Kingdom) with variable, atypical nonexertional chest and interscapular pain associated with ectopic beats. She was hypertensive and had a family history of ischemic heart disease but not of connective tissue disorders. An electrocardiogram showed sinus rhythm with ectopic beats and evidence of left ventricular hypertrophy. A transthoracic echocardiogram revealed a hypertrophied, well-contracting left ventricle, marked left atrial dilation and a large cavity straddling the atrioventricular groove (Figure 1). This was subsequently demonstrated on cardiac magnetic resonance imaging to be a large, $7 \mathrm{~cm}$ aneurysm of the left coronary sinus of Valsava (Figure 2 and Video 1). Biventricular size and function were normal and there was no aortic regurgitation. Venereal Disease Research Laboratory serology was negative.

Her case was reviewed at a multidisciplinary team meeting; surgery was deemed to be appropriate on prognostic grounds. Computed tomography (CT) coronary angiography was performed before surgery. The coronary calcium score was zero, and the circumflex and right coronary arteries were unobstructed. The ostium of the left main stem was observed to be displaced superiorly by the aneurysm sac (not arising from within the sac), and the coronary artery was stretched over the $7 \mathrm{~cm}$ aneurysm. The calibre of this portion of the left coronary artery appeared to be small and coronary flow appeared sluggish (ie, it enhanced poorly) in this section; distally, it was observed to be unobstructed.

During surgery, the dilated left coronary sinus of Valsava manifested as a prominent swelling lateral and posterior to the main pulmonary artery, with epicardial coronary arteries visibly stretched over the sac. There was a large cavity from the left coronary sinus proximal to the coronary ostium with effacement of the aortic wall and displacement of the left coronary ostium to the level of the sinotubular junction. The aortic valve annulus was very thin. In view of these observations, it was deemed to be necessary to proceed to an aortic valve and root replacement. Her aortic valve was replaced using a $23 \mathrm{~mm}$ Magna Ease progressive reduction in systemic arterial pressure and cardiac output disappeared, culminating in ventricular fibrillation, which was believed to be due to ischemia induced by the stretching of the native coronary arteries over the roof of the aneurysm sac. Therefore, the patient underwent coronary artery bypass grafting with revascularization of the left-sided system. The authors suggest that preoperative assessment, including noninvasive assessment of myocardial ischaemia, should be part of the work-up.

Key Words: CABG; Coronary ischemia; Left sinus of Valsalva aneurysm; Surgical technique

3300TFX pericardial tissue valve (Edwards Lifesciences, USA), which was implanted with horizontal mattress sutures placed from the ventricular aspect of the native annulus. The sutures were then passed from the inside to the outside of a $26 \mathrm{~mm}$ Hemashield Platinum tube graft. The valve seated well, and with the tube graft in place all sutures were ligated. Left and right coronary ostia were raised on buttons of native aortic tissue, holes were cut in the posterior and anterior aspect of the tube graft and the ostial buttons were sutured in place on the internal aspect of the tube graft.

Intraoperatively and while preparations were made for chest closure, there was a progressive reduction in systemic arterial pressure and cardiac output disappeared, culminating in ventricular fibrillation. Subsequently, revascularization of the left-sided system with vein grafts to the left anterior descending, intermediate and circumflex arteries was performed, and the patient was successfully weaned from bypass and discharged from hospital without subsequent sequelae. Six months later, a CT aortogram confirmed three patent vein grafts (distal left anterior descending, first diagonal branch and first obtuse marginal) as well as patent native coronary arteries arising from the coronary buttons. The scan was performed due to concomitant pulmonary nodules that were noted preoperatively, which interval surveillance has subsequently determined to be nonmalignant.

\section{DISCUSSION}

Sinus of Valsava aneurysms (SVAs) are rare. Nintey-four percent affect the right coronary sinus, $5 \%$ the noncoronary sinus and only $1 \%$ the left sinus (1). Congenital etiology is more common (associated with atrial septal defect, ventricular septal defect, and aortic or pulmonary valve abnormalities); acquired SVA may be due to bacterial endocarditis, syphilis, atherosclerosis, trauma, connective tissue disorder (eg, Marfan syndrome) and senile-type dilation. (2). Patients with unruptured SVAs are mostly asymptomatic, but patients may report nonspecific complaints such as dyspnea, fatigue, chest pain and palpitations. Echocardiography, contrast aortography, multislice spiral CT, cardiac catheterization and magnetic resonance imaging are used in the diagnosis of SVAs.

Department of Cardiology, Norfolk and Norwich University Hospital NHS Foundation Trust, Norwich, United Kingdom

Correspondence: Dr Panagiota Anna Chousou, 12 Chatsworth Avenue, Cambridge CB4 3LT, United Kingdom. Telephone 44-77-95-962-009,

fax 44-16-03-289-666, e-mail pachousou@hotmail.com 


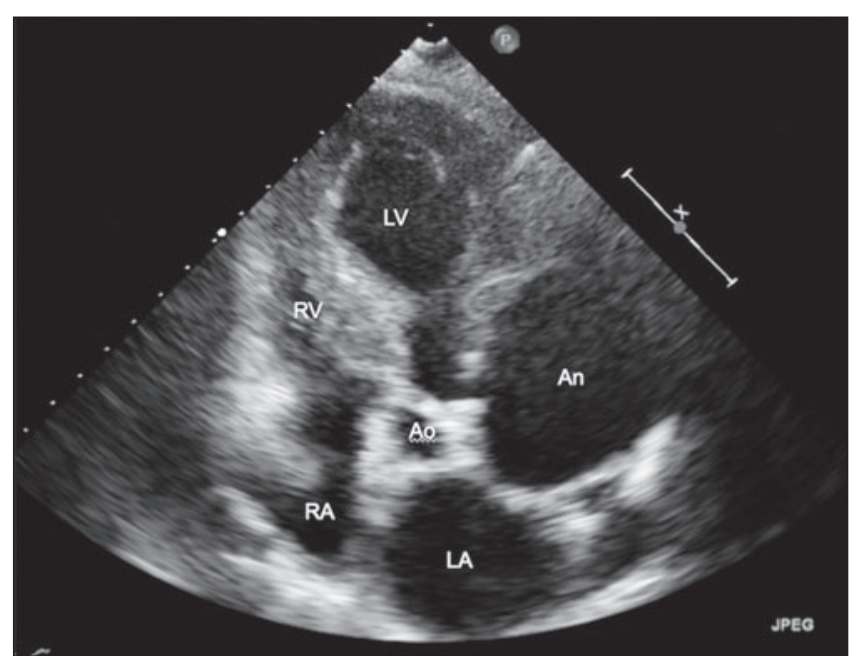

Figure 1) Transthoracic echocardiogram of five-chamber apical view showing a large cavity that appears to straddle the atrioventricular groove. An Aneurysm, Ao Aorta; LA Left atrium; LV Left ventricle; RA Right atrium; RV Right ventricle

Repair is generally advocated for either large unruptured aneurysms encroaching on nearby structures or aneurysms having the potential to rupture due to increasing size on surveillance imaging. The first successful SVA surgery (congenital) was only reported in 1976 and the surgical approach for treatment has been influenced by case reports. In isolated, unruptured SVAs without compromise of the aortic valve, repair may be accomplished by simple plication or excision of the aneurysm and patch closure of the defects. Joshi et al (3) previously reported two cases involving modified root reconstruction in this situation. Prosthetic aortic root replacement and reimplantation of the coronary arteries may be performed in patients with a dilated annulus and multiple sinus involvement (4) or, as in this case, due to the thinned aortic root, which is believed to represent senile-type degeneration. In cases associated with aortic regurgitation, aortic valve replacement or valvuloplasty may be required.

Lijoi et al (1) described a patient (congenital etiology) with a thrombus-filled left SVA causing direct compression of the coronary artery producing ischemia on exercise testing, which was treated only with isolated exclusion of the neck of the aneurysm (subsequent negative stress thallium testing). Their review of 19 other cases presenting

\section{REFERENCES}

1. Lijoi A, Parodi E, Passerone GC, et al. Unruptured aneurysm of the left sinus of Valsalva causing coronary insufficiency. Tex Heart Inst J 2002;29:40-4.

2. Goldberg N, Krasnow N. Sinus of Valsalva aneurysms. Clin Cardiol 1990;13:831-6.

3. Joshi P, Garlick B, Dunning J. Modified root reconstruction methods to treat unruptured sinus of Valsalva aneurysm (UASOV) - 2 case reports. Heart Lung Circ 2006;15:389-92

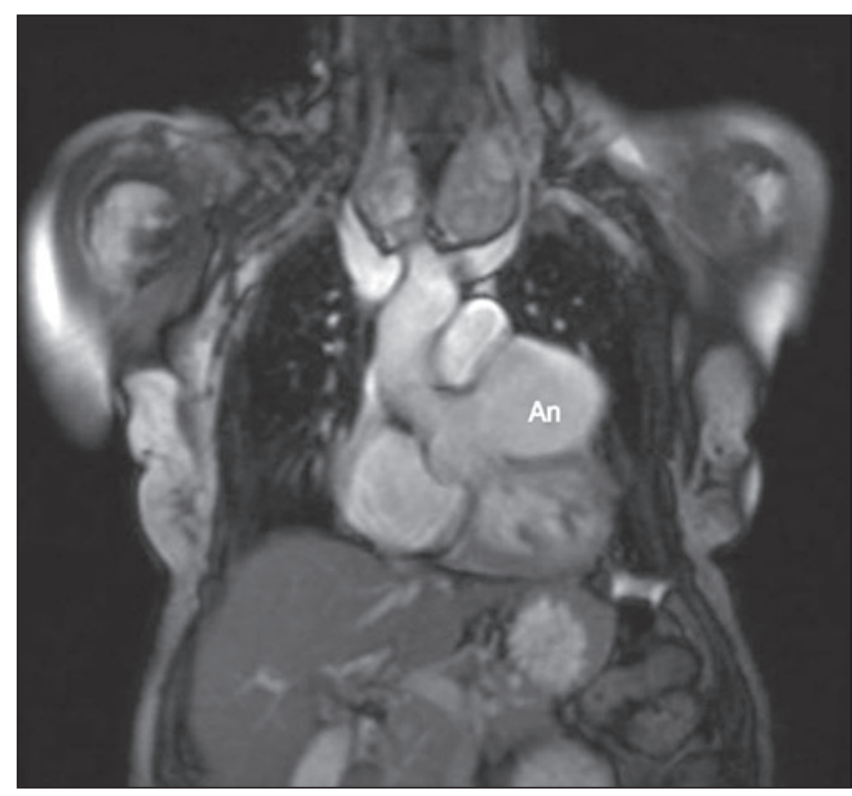

Figure 2) Cardiac magnetic resonance imaging showing a grossly aneurysmal left coronary sinus measuring $7 \mathrm{~cm}$ in diameter. An Aneurysm

with angina or myocardial infarction with unruptured SVA found one other case (congenital) in which isolated closure of the aneurysm was effective. They propose that isolated SVA exclusion or repair may be appropriate, thus avoiding additional risks associated with coronary artery bypass grafting. That may be true in congenital SVA but, as demonstrated in the present case, may be unsuccessful. Indeed, Dias et al (5) subsequently reported a case in which perioperative ischemia necessitated an unplanned concomitant coronary artery bypass graft.

Our patient's initial symptoms were atypical for myocardial ischemia, and surgery was planned to avoid coronary artery bypass grafting. However, revascularization of the left-sided system was required due to perioperative ischemia pertaining to the stretched left main stem. A reflection for clinicians who encounter a case that is not congenital or associated with infection is to consider myocardial perfusion imaging in the diagnostic work up to aid operative planning particularly because the senile-degenerative SVA may be more commonly observed in the present era.

4. Mourad F, Tang A. Sinus of Valsalva aneurysm in Blau's syndrome. J Cardiothoracic Surg 2010;5:16.

5. Dias RR, Camurça FD, Filho OA, et al. Right sinus of Valsalva aneurysm causing extrinsic coronary compression. Arq Bras Cardiol 2009;92:e47-50. 